Onkologe $2022 \cdot 28: 248-252$

https://doi.org/10.1007/s00761-022-01096-w

Angenommen: 10. Januar 2022

Online publiziert: 28. Januar 2022

(C) The Author(s), under exclusive licence to Springer Medizin Verlag GmbH, ein Teil von Springer Nature 2022

\section{Fast zwei Jahre Coronapandemie aus der Perspektive von Krebsbetroffenen}

\author{
Andrea Gaisser' $\cdot$ Rachel D. Eckford ${ }^{2}$. Volker Arndt ${ }^{3,4} \cdot$ Daniela Doege ${ }^{3} \cdot$ Evelyn Kludt $^{1}$. \\ Jasper Ubels ${ }^{2} \cdot$ Michael Schlander ${ }^{2} \cdot$ Susanne Weg-Remers ${ }^{1}$ \\ 'Krebsinformationsdienst, Deutsches Krebsforschungszentrum (DKFZ), Heidelberg, Deutschland \\ ${ }^{2}$ Abteilung für Gesundheitsökonomie, Deutsches Krebsforschungszentrum (DKFZ), Heidelberg, \\ Deutschland \\ ${ }^{3}$ Arbeitsgruppe Cancer Survivorship, Abteilung für Klinische Epidemiologie und Alternsforschung, \\ Deutsches Krebsforschungszentrum (DKFZ), Heidelberg, Deutschland \\ ${ }^{4}$ Epidemiologisches Krebsregister Baden-Württemberg, Deutsches Krebsforschungszentrum (DKFZ), \\ Heidelberg, Deutschland
}

Im zweiten Pandemiewinter scheinen sich die Ereignisse des vorangegangenen Jahres zu wiederholen. Trotz Verfügbarkeit wirksamer Impfstoffe sind die Infektionszahlen höher denn je, erneut gerät die Intensivmedizin an Kapazitätsgrenzen. Den Krebsinformationsdienst des Deutschen Krebsforschungszentrums erreichten besonders im Frühjahr 2020 Tausende von Anfragen verunsicherter und besorgter Betroffener und Angehöriger. Es gab hier wie auch aus einem Monitoring bei 18 deutschen Comprehensive Cancer Centers Hinweise auf Einschränkungen in der onkologischen Versorgung. Ab Sommer 2020 ging deshalb eine Befragungsstudie gezielt den Auswirkungen der Pandemie für Krebsbetroffene nach.

Krebskranke zählen zu den durch die Coronavirus-Erkrankung-2019 (COVID-19) besonders gefährdeten Gruppen mit deutlich erhöhtem Risiko für einen schweren Verlauf. Entsprechend war bei ihnen die Verunsicherung insbesondere in der ersten Zeit der Pandemie groß, wie die Anfragen an den Krebsinformationsdienst eindrucksvoll zeigten.

Auch auf die onkologische Versorgung hatte die Fokussierung auf Corona und die „Reservierung" von Ressourcen für die Versorgung der schwer an COVID-19 Erkrankten Auswirkungen: Nicht dringend erforderliche Untersuchungen, teilweise auch
Behandlungen wurden ggf. verschoben, Nachsorgetermine ausgesetzt, um einerseits Kapazitäten freizuhalten und andererseits Krebspatienten nicht unnötig durch Aufsuchen von Praxis oder Klinik zu gefährden. Umgekehrt vermieden viele Betroffene aus Furcht vor Ansteckung von sich aus Arztbesuche. Ebenfalls von dieser Vermeidungstendenz betroffen waren Untersuchungen zur Krebsfrüherkennung und zur Abklärung von Symptomen. Eine vom Deutschen Krebsforschungszentrum, der Deutschen Krebshilfe und der Deutschen Krebsgesellschaft gebildete "TaskForce" zur Beobachtung der Versorgungslage warnte nach entsprechenden Berichten aus der Ärzteschaft im Mai 2020 davor, solche Diagnostik aufzuschieben - es könne sich eine Bugwelle von zu spät diagnostizierten Krebsfällen aufbauen [1]. Ob und ggf. welche Folgen dies hat, wird sich erst noch zeigen.

Zum Monitoring der onkologischen Versorgungskapazität und zur Identifizierung von Engpässen initiierte die TaskForce im April 2020 eine prospektive PanelStudie mit Beteiligung von 18 Comprehensive Cancer Centers (CCC) in Deutschland, die zusammen 15-20\% aller jährlich an Krebs neu Erkrankenden betreuen. Diese Zentren wurden seither regelmäßig zur Situation befragt, zunächst engmaschig, dann in größeren Abständen. Erste Ergebnisse wurden bereits Ende 2020 veröffentlicht [2]. Sie zeigten, dass nach 


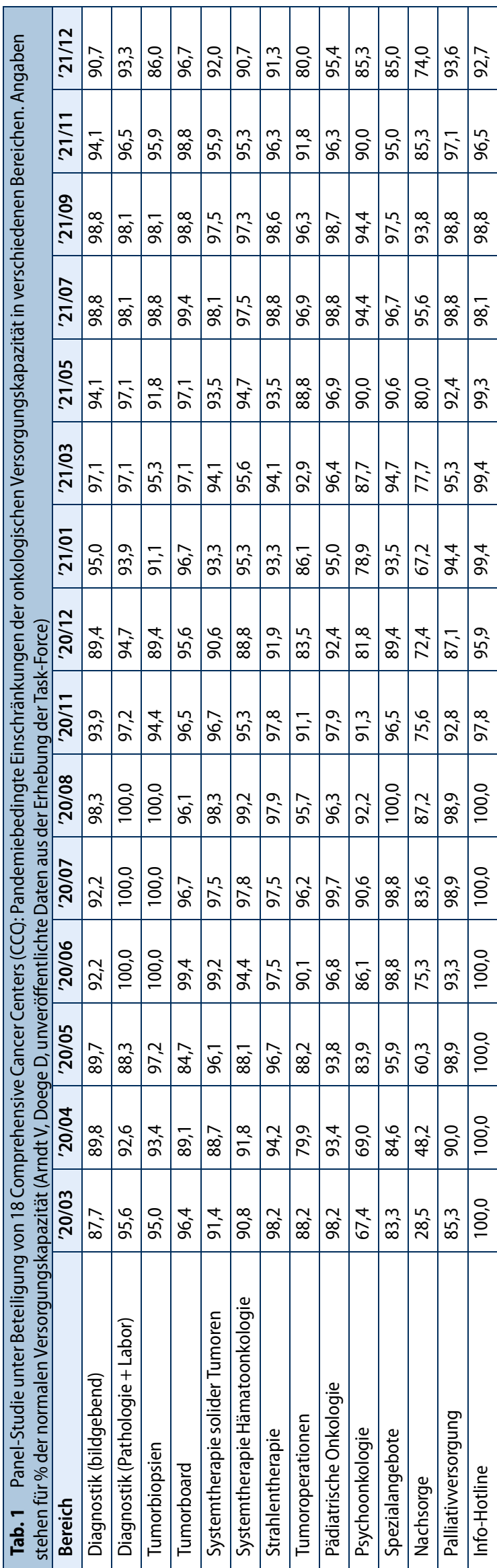

Abklingen der ersten Welle die onkologische Primärversorgung zunächst wieder weitgehend unbeeinträchtigt war, bevor es im Dezember 2020 und im Januar 2021 erneut zu Einschränkungen kam, wenn auch weniger ausgeprägt. In den Bereichen Nachsorge, psychoonkologische Betreuung und Beratung war die Kapazität allerdings längerfristig reduziert. Die Befragung wird weitergeführt. Im Dezember 2021 zeichnen sich erneut Engpässe $a b$, insbesondere im intensivmedizinischen Bereich ( $\bullet$ Tab. 1). Aktuell berichten zwei Drittel der befragten Zentren von einer drohenden oder latent vorhandenen Triage. In der Folge könnten aber auch Patienten mit anderen schweren Erkrankungen unter der Situation leiden, wenn Personal aus dem Stationsbetrieb zur Versorgung von COVID-19-Patienten abgestellt werden muss [3].

\section{Hoher Informations- und Beratungsbedarf}

Die tatsächlichen oder befürchteten Konsequenzen und Auswirkungen der Pandemie für Krebsbetroffene waren Thema einer Vielzahl von Anfragen an den Krebsinformationsdienst, die ab April 2020 gesondert dokumentiert wurden. Die Zahlen folgten dem Verlauf der Pandemie mit einer ersten Spitze während der ersten Welle, als die Unsicherheit noch besonders groß war, einem deutlichen Abebben im Sommer und erneutem Anstieg mit Beginn der zweiten Welle ab November 2020 und insbesondere mit dem angekündigten Beginn der Impfkampagne.

Während zunächst besonders die Gefährdung von Krebspatienten durch COVID-19, das Leben mit Krebs in der Pandemie und psychosoziale Themen im Vordergrund standen, betrafen die Anfragen ab Dezember 2020, nach ersten Nachrichten über die bevorstehende $\mathrm{Zu}$ lassung von Impfstoffen, zunehmend die Impfung gegen SARS-CoV-2, vor allem den Zugang dazu und die Wirksamkeit und Sicherheit der Vakzine bei Krebspatienten. Betroffene suchten konkrete Informationen und Handlungsempfehlungen für ihre individuelle Situation. Dieses Thema dominierte im weiteren Verlauf vor allen anderen. Auch hier bot der Krebsinformationsdienst Informationen 
3.000

2.500

2.000

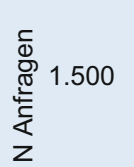

Z

1.000

500

0

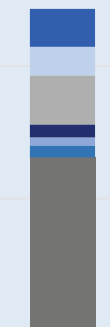

Welle 1 Apr-Mai 20
Ruhephase Jun-0kt 2020

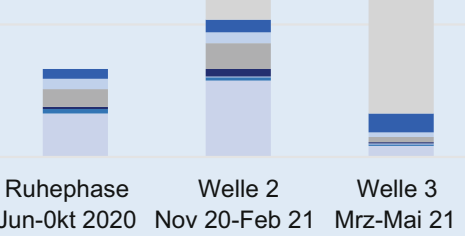

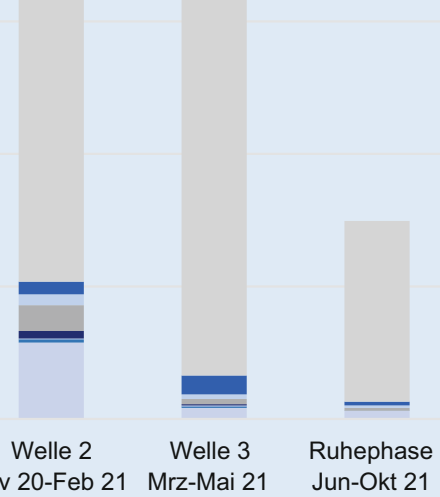

Impfung

- Sozialrechtliche Aspekte Krebs und COVID-19

Psychosoziale Aspekte Krebs und COVID-19

Leben mit Krebs und COVID-19

- Behandlung verschoben/abgesagt

- Behandlung geändert

- Untersuchungen verschoben/abgesagt

Welle 4

ab Nov 21

Abb. 1 A Anfragen zu SARS-CoV-2 und COVID-19 und Inhalte nach Pandemiephase (April 2020 bis November 2021; $n=6776$ )

nach dem jeweils aktuellsten Stand des Wissens per Telefon, E-Mail und auf der Website. Die Verteilung der Anfragen und Themen über die bisherigen 4 Wellen der Pandemie zeigt $\bullet$ Abb. 1.

\section{Pandemieerfahrungen von Krebsbetroffenen - eine Befragungsstudie}

Der in den vielen Anfragen an den Krebsinformationsdienst erkennbare hohe Informationsbedarf, die Besorgnis und Verunsicherung wie auch die Meldungen zu Einschränkungen und Engpässen in der onkologischen Versorgung waren Anlass für eine Befragungsstudie: Welche Folgen hat oder hatte die Pandemie für Patientinnen und Patienten? Welche Belastungen erleben sie [4]?

Die Studie konnte am 11. Juli 2020 nach Genehmigung durch die Ethikkommission der Universität Heidelberg starten.

Ein gemeinsam mit der Abteilung Gesundheitsökonomie am Deutschen Krebsforschungszentrum und weiteren Wissenschaftlern aus Versorgungsforschung und Medizinethik entwickelter Fragebogen sollte die Erfahrungen Betroffener genauer beleuchten:
- Hat sich durch die Pandemie etwas in der onkologischen Versorgung und Betreuung geändert?

- Welche Sorgen und Belastungen spüren die Betroffenen, und wie ist ihre psychische Verfassung?

- Haben sie durch die Coronapandemie besondere finanzielle Belastungen oder Einbußen?

Zu der anonymen Online-Befragung wurden Betroffene eingeladen, die sich an den E-Mail-Service des Krebsinformationsdiensts wandten. Die Erhebung wurde am 30. Juni 2021 mit 621 auswertbaren Rückläufen abgeschlossen. Sie deckt damit die „Ruhephase" nach der ersten Welle sowie die zweite und dritte Welle der Pandemie ab.

Drei Viertel der Teilnehmerinnen und Teilnehmer (76\%) waren Frauen, das mediane Alter betrug 60 Jahre. Brustkrebs war als Erkrankung mit 50\% am häufigsten vertreten. Mit $64 \%$ Hochschulreife war der Bildungsstand hoch. Rund ein Drittel (32\%) befand sich nach Diagnosestellung und während der Primärtherapie, bei $40 \%$ war die erste Behandlung abgeschlossen. Damit ist das Kollektiv demjenigen der Nutzer des Krebsinformationsdiensts insge- samt weitgehend vergleichbar, allerdings nicht bevölkerungsrepräsentativ.

\section{Auswirkungen auf die onkologische Versorgung}

Rund $13 \%$ der Befragten berichteten von Auswirkungen auf ihre Versorgung, vor allem in der ersten Phase der Pandemie. Dabei erhielt die überwiegende Mehrzahl (96\%) ihre Antitumortherapie wie geplant. Aber es gab durchaus Änderungen, vor allem bei systemischen Therapien, aber auch bei Operationen, meist im Sinne von Verschiebungen. Kontroll- und Nachsorgetermine wie auch die psychoonkologische Versorgung waren deutlich häufiger von Einschränkungen - ebenfalls meist Terminverschiebungen oder Umstellung der Kontakte auf Telefon oder Video - betroffen, hauptsächlich, aber nicht nur, während der ersten Erhebungsphase. Es ist anzunehmen, dass einige Befragte hier retrospektive Angaben machten. Als einziger statistisch signifikanter prädiktiver Faktor für Änderungen in der Versorgung ergab sich in der Analyse die Phase der Erkrankung: Nach Abschluss der Primärtherapie wurden mehr Änderungen berichtet als in anderen Behandlungsphasen. Für alle 


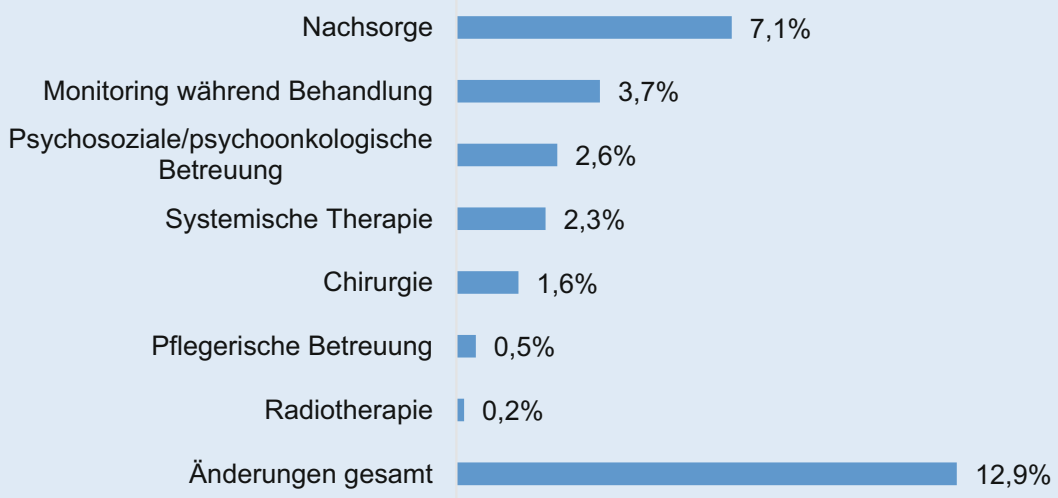

Abb. $2 \Delta$ Art und Häufigkeit von Änderungen bei Behandlung und Versorgung ( $n=621$; Mehrfachnennungen möglich)

weiteren untersuchten Faktoren wie Alter, Geschlecht, Bildung, Tumorart, Versicherungsstatus und Pandemiephase zeigte sich kein Zusammenhang. Abb. 2 zeigt Art und Häufigkeit von Änderungen.

\section{Pandemiesorgen}

Ein beträchtlicher Teil der Studienteilnehmer - 33\% - machte sich Sorgen über die Qualität ihrer medizinischen Versorgung unter Pandemiebedingungen. 30\% hatten Angst, selbst an COVID-19 zu erkranken oder daran zu versterben, und mehr noch um Angehörige und Freunde. Hier zeigte sich in der dritten Welle ein deutlicher Rückgang - die Impfkampagne war zu diesem Zeitpunkt schon länger angelaufen. Noch mehr machten vielen Betroffenen die Beschränkungen persönlicher Kontakte in Lockdownphasen zu schaffen, ganz besonders mit Freunden und Familie und in der Öffentlichkeit. Nur rund $20 \%$ gaben an, sich durch die Situation gar nicht beeinträchtigt zu fühlen. Frauen litten stärker unter den Kontaktbeschränkungen als Männer (76 vs. $54 \%$ ).

Thema war auch, inwieweit die Befragten finanzielle Sorgen und Belastungen durch die Pandemie erfuhren. Jeder Fünfte gab an, zusätzliche Ausgaben für die Gesundheitsversorgung zu haben, vor allem für Masken und Desinfektionsmittel, überwiegend unter $200 €$ pro Monat - wobei zwei Drittel bereits Mehrausgaben durch ihre Krebserkrankung bejahten: etwa für Zuzahlungen, Fahrtkosten und nicht erstattungsfähige Medikamente oder The- rapien. $17 \%$ machten sich Sorgen um ihre Arbeitsstelle und mögliche finanzielle Folgen, und $15 \%$ hatten bereits Einkommenseinbußen durch Kurzarbeit oder Jobverlust, die sich überwiegend im Bereich bis $800 €$ monatlich bewegten, teilweise aber auch deutlich darüber. Die Ergebnisse zu den einzelnen Fragen zeigt - Abb. 3 im Überblick.

\section{Hohes Level von Angst- und depressiven Symptomen}

Zur Erfassung psychischer Belastung war die Hospital Anxiety and Depression Scale (HADS) in den Fragebogen integriert. Jeweils 7 Items dienen der Einschätzung von Angstsymptomen (HADS-A) und Depressivität (HADS-D). Ein Summenwert zwischen 8 und 11 spricht jeweils für grenzwertige Symptomatik, ab 11 geht man von einer manifesten Störung aus.

Unter Einschluss von Grenzfällen lag die Prävalenz von Angstsymptomen bei $54,6 \%$ bzw. bei 38,8\% für Depression. Als symptomatisch geltende Werte $\geq 11$ zeigten 30 bzw. 21,3\% der Befragten. Der mittlere Score für Angstsymptome betrug 8,2, für Depressivität 6,8. Mit einem HADS-A-Score von 8 und höher statistisch signifikant assoziierte Faktoren waren Änderungen in der Versorgung, Angst vor eigener Erkrankung an COVID-19 sowie dass Angehörige und Freunde erkranken oder sterben könnten, und Bedenken, die Pandemiesituation könnte die Qualität der medizinischen Versorgung beeinträchtigen $(p<0,001)$. Bei Berufstätigen und bei
Personen mit geringerer Bildung war die Prävalenz von Angstsymptomen ebenfalls erhöht. Als prädiktiv für depressive Symptome (HADS-D) ergab die multivariate Analyse ebenfalls Änderungen in der Versorgung und Bedenken hinsichtlich der Versorgungsqualität, außerdem die Sorge, nahestehende Personen könnten erkranken $(p<0,001)$. Die Scorewerte blieben über den gesamten Befragungszeitraum vergleichbar erhöht.

\section{Belastungen erkennen und Unterstützung gewährleisten}

Im Vergleich zur Mehrzahl der bisher publizierten Studien zu diesen Fragestellungen deckt die Erhebung einen langen Zeitraum der Pandemie ab. Auch wenn das Kollektiv nicht repräsentativ ist, lieferte sie „Real-lifeDaten" zu den Auswirkungen im Verlauf eines Jahrs aus der Betroffenenperspektive. Die onkologische Versorgung war demnach überwiegend gewährleistet, die psychischen und psychosozialen Belastungen blieben allerdings über die gesamte Befragungsdauer ausgeprägt. Die Prävalenz von Angst- und depressiven Symptomen war in dieser Studie höher als in präpandemischen Studien mit Krebsbetroffenen und auch im Vergleich zu HADS-Studien, die in den ersten Pandemiephasen durchgeführt wurden. Zudem wurde deutlich, dass die Pandemie auch die ökonomische Situation vieler Patientinnen und Patienten verschlechtert hat und sie in Sorge versetzt. An Krebs Erkrankte sind eine in vielerlei Hinsicht vulnerable Gruppe. Daher ist es wichtig, Belastungen und entsprechende Risikofaktoren zu identifizieren und entsprechende Unterstützungsangebote bedarfsgerecht und niederschwellig zur Verfügung zu stellen.

\section{Von der Pilotstudie zur systematischen Erhebung}

Im Mai 2021, kurz vor dem Ende der Pilotbefragung, startete am Deutschen Krebsforschungszentrum (DKFZ) die CroKuSStudie (Folgen der Corona-Beschränkungen für Krebstherapie und Survivorship). Der zuvor verwendete Fragebogen wurde für diese Erhebung erweitert, und die Rekrutierung erfolgte über das Epidemiologische Krebsregister Baden-Württem- 


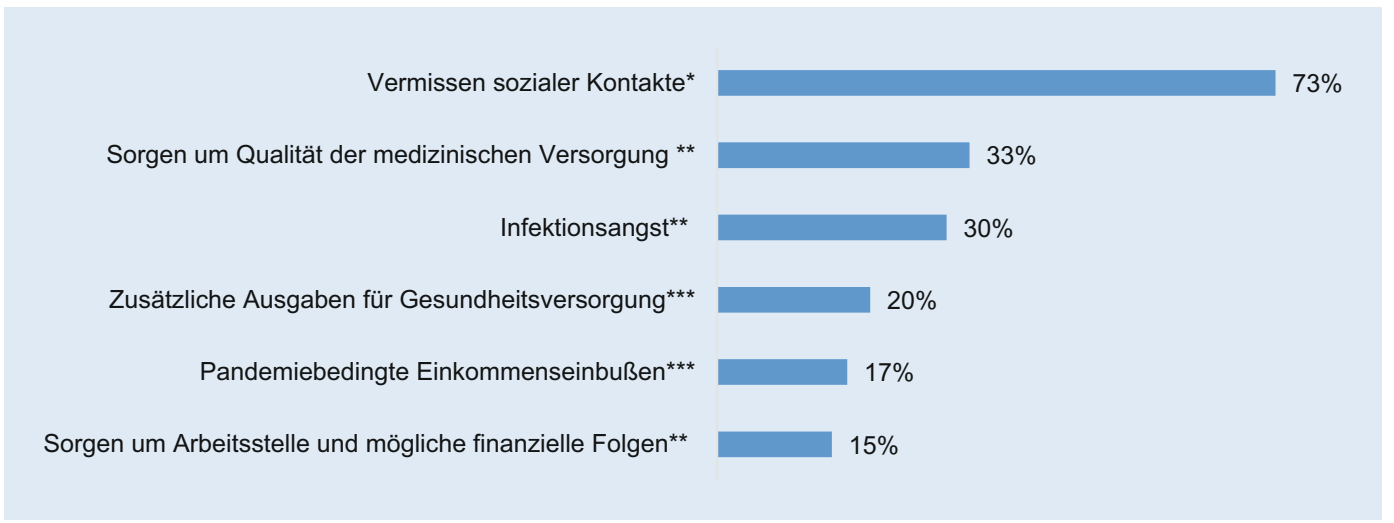

Abb. $3 \triangleleft$ Belastungen und Sorgen durch die Pandemie (6 Einzelitems; *sehr/ ziemlich, ${ }^{* *}$ Zustimmung/ Vollzustimmung, ${ }^{* * *}$ ja)

berg $[5,6]$. Dort erfasste Patientinnen und Patienten mit Brustkrebs, Darmkrebs, Lungenkrebs, Prostatakrebs und hämatoonkologischen Erkrankungen wurden zur Teilnahme eingeladen. Themen sind auch hier pandemiebedingte Veränderungen der Versorgung, psychosoziale Folgen sowie Auswirkungen auf die gesundheitsbezogene Lebensqualität und die finanzielle Situation. Verglichen werden kurz vor der Pandemie an Krebs erkrankte Patienten und solche, deren Diagnose und Behandlung schon länger zurückliegen ("cancer survivors").

Die geplante Teilnehmerzahl von 2400 ist mittlerweile bereits überschritten, und die Rekrutierung steht kurz vor ihrem Ende. Bei allen Entitäten sind die Rückläufe auch für entsprechende Subgruppenanalysen ausreichend. Persönliche Rückmeldungen und Kommentare von Teilnehmenden lassen erkennen, dass die Pandemie sehr unterschiedlich erlebt wurde von gar keinen Einschränkungen bis hin zu Mehrfachbelastungen, wie etwa bei zusätzlichem Arbeitsplatzverlust. Die Auswertung ist im Gang. Aus bisher plausibilisierten Datensätzen geht hervor, dass auch hier ungefähr 10-15\% der Befragten Änderungen in ihrer Krebsversorgung erfahren haben. Diese Änderungen betrafen das gesamte Spektrum, von der Durchführung einzelner Nachsorgetermine per Video oder Telefon bis hin zu abgesagten Operationen.

Mehr Wissen zu den in den beiden Studien untersuchten Aspekten kann spezifische Unterstützungsbedarfe von Krebspatientinnen und -patienten aufdecken und dazu beitragen, dass Menschen mit Krebs und anderen Erkrankungen auch in Zeiten wie der "Coronakrise" eine bedarfsge- rechte, qualitativ hochwertige Versorgung erfahren.

\section{Korrespondenzadresse}

\section{Andrea Gaisser}

Krebsinformationsdienst, Deutsches

Krebsforschungszentrum (DKFZ)

Im Neuenheimer Feld 280, 69120 Heidelberg, Deutschland

a.gaisser@dkfz.de

\section{Einhaltung ethischer Richtlinien}

Interessenkonflikt. A. Gaisser, R.D. Eckford, V. Arndt, D. Doege, E. Kludt, J. Ubels, M. Schlander und S. WegRemers geben an, dass kein Interessenkonflikt besteht.

Für diesen Beitrag wurden von den Autoren keine Studien an Menschen oder Tieren durchgeführt. Für die aufgeführten Studien gelten die jeweils dort angegebenen ethischen Richtlinien.

\section{Literatur}

1. Deutsches Krebsforschungszentrum, Deutsche Krebshilfe, Deutsche Krebsgesellschaft (2020) Corona Task Force warnt weiterhin vor zu spät diagnostizierten Krebserkrankungen. Gemeinsame Pressemitteilung 14.05.2020. https:// www.dkfz.de/de/presse/pressemitteilungen/ 2020/dkfz-pm-20-31-Corona-Task-Forcewarnt-weiterhin-vor-zu-spaet-diagnostiziertenKrebserkrankungen.php. Zugegriffen: 20. Dez. 2021

2. Fröhling S, Arndt V (2020) Versorgung von Krebspatienten: Corona-Effekt in der Onkologie. Dtsch Arztebl 117(46):A-2234/B-1893

3. Deutsches Krebsforschungszentrum, Deutsche Krebshilfe, Deutsche Krebsgesellschaft (2021) Universitätskliniken fürchten Triage bei Krebspatienten. Gemeinsame Pressemitteilung 21.12.2021. https://www.dkfz.de/de/ presse/pressemitteilungen/2021/dkfz-pm-2172-Universitaetskliniken-fuerchten-Triage-beiKrebspatienten.php.Zugegriffen:21.Dez. 2021

4. EckfordREetal (2021) The COVID-19 pandemic and cancer patients in Germany: impact on treatment, follow-up care and psychological burden. Front Public Health
5. Krebsregister Baden-Württemberg (2021) Melderanschreiben Nr. 3, März 2021: Forschungsvorhaben. https://www.krebsregister-bw.de/home/ service-downloads/nl3\#c4985. Zugegriffen: 20. Dez. 2021

6. Deutsches Krebsforschungszentrum (2021) DKFZ startet Studie zu psychosozialen Belastungen von Krebspatienten während der Corona-Pandemie. Pressemitteilung vom 06.04.2021. https://www.dkfz.de/de/presse/ pressemitteilungen/2021/dkfz-pm-21-21-DKFZstartet-Studie-zu-psychosozialen-Belastungenvon-Krebspatienten-waehrend-der-CoronaPandemie.php.Zugegriffen: 20.Dez. 2021 